\title{
Les femmes au foyer
}

Des activités méconnues et peu valorisées en employabilité

Housewives and links with the work and the labor market

Anne-Marie Dieu, Christine Delhaye et Annie Cornet

\section{(2) OpenEdition}

\section{Journals}

Édition électronique

URL : https://journals.openedition.org/travailemploi/1810

DOI : 10.4000/travailemploi.1810

ISSN : 1775-416X

Éditeur

DARES - Ministère du Travail

Édition imprimée

Date de publication : 15 juin 2010

Pagination : 27-38

ISSN : 0224-4365

Référence électronique

Anne-Marie Dieu, Christine Delhaye et Annie Cornet, « Les femmes au foyer », Travail et Emploi [En ligne], 122 I avril-juin 2010, mis en ligne le 15 juin 2012, consulté le 15 février 2023. URL : http:// journals.openedition.org/travailemploi/1810; DOI : https://doi.org/10.4000/travailemploi.1810 


\title{
Les femmes au foyer: des activités méconnues et peu valorisées en employabilité
}

\author{
Anne-Marie Dieu (*), Christine Delhaye (**), Annie Cornet $(* * *)$
}

Cet article s'intéresse aux femmes au foyer. En s'appuyant sur une recherche qualitative, réalisée en Wallonie (Belgique francophone), les auteures mettent en évidence que le rapport au travail et au marché de l'emploi des femmes dites «inactives» est bien plus complexe que les représentations communes ne le laissent apparaître. Outre les activités familiales et parentales, les femmes au foyer développent, lors de leur période de vie "au foyer», une série d'activités productives, créatives et de service qui sortent de la sphère familiale et qui contribuent à leur procurer une série de gratifications sociales, psychologiques et symboliques, tout en leur assurant des revenus occasionnels ou plus réguliers. Ce rapport occasionnel avec des activités productives leur permet de développer une série de compétences dont elles ne sont pas toujours conscientes elles-mêmes alors qu'elles pourraient être mobilisées pour faciliter leur retour sur le marché du travail et donc améliorer leur employabilité.

Il peut sembler étonnant de proposer un article sur les femmes au foyer dans une revue consacrée au travail et à l'emploi. En effet, les femmes au foyer ne sont, a priori, pas concernées par le marché du travail. Elles sont d'ailleurs classées dans les statistiques dans la catégorie des «inactives». Si de fait elles sont sans emploi, pendant parfois de longues périodes, notre recherche vise à comprendre comment elles gèrent leur retour sur le marché du travail. Nous nous sommes donc intéressées aux «femmes rentrantes», pour reprendre l'appellation de VAN REGENMORTEL, De Cock et Vandeloo (1990). Nous avons ciblé des femmes qui avaient cessé pendant plus de deux ans de travailler pour des raisons familiales et qui souhaitaient ou avaient réintégré le marché du travail. En nous fondant sur une revue de la littérature, nous pensions initialement que leur carrière professionnelle était comme «saucissonnée», avec une période d'activité professionnelle «avant» le retrait et une seconde période professionnelle «après» ce retrait. L'analyse des interviews réalisées a montré que le rapport au marché du travail et au marché de l'emploi des femmes dites «inactives» est bien plus complexe, avec des retraits «par étapes», des retours occasionnels et irréguliers et des activités en dehors du cercle familial, nombreuses et variées. Ceci nous a conduit à parler d'un rapport en pointillé au marché $d u$ travail pour ces femmes. Les femmes au foyer d'aujourd'hui sont des femmes qui déplacent les frontières du travail non déclaré, du travail déclaré et des activités au foyer. Ce sont des équilibristes, qui parta-

(*) CESEP, collaboratrice scientifique EGID, HEC-ULg, école de gestion de l'université de Liège; am.dieu@ulg.ac.be.

(**) Communauté Française de Belgique, collaboratrice scientifique EGID - Etudes sur le genre et la diversité en gestion, université de Liège, Belgique ; christine.delhaye@cfwb.be (***) Annie Cornet, professeure ordinaire (full professor), Hec-Ulg, directrice d'EGiD, Etudes sur le Genre et la Diversité en gestion, université de Liège, Belgique ; annie.cornet@ulg.ac.be. gent le destin de nombreuses personnes aujourd'hui, entre deux statuts, deux emplois ou deux modes de vie. Cet article vise à documenter ces activités qui sont largement invisibles et se traduisent rarement, pour les femmes et les acteurs de l'accompagnement sur le marché du travail, par des compétences valorisables dans les parcours professionnels.

\section{Revue de littérature: un rapport à l'emploi “en pointillé» méconnu}

Décider de se retirer du marché du travail n'est pas sans conséquence en termes d'identité et de statut. Comme le font remarquer BAUdELOT et GollaC, dans leur ouvrage Travailler pour être heureux? (2003), les personnes, dans la société actuelle, se définissent avant tout par leur statut professionnel, au point que ce que nous faisons comme métier devient par extension ce que nous sommes, avec pour effet que beaucoup s'identifient en nommant leur métier. Nous sommes dans une société où les femmes au foyer sont de moins en moins considérées (MAISON, 2007). Dominique Maison, dans la thèse qu'elle leur a consacrée, qualifie leur statut de «déviant» par rapport à la norme actuelle. Opter pour ce statut, c'est aussi prendre un risque important, en raison de l'augmentation du nombre de divorces et de séparations des couples et de la difficulté de retourner sur le marché du travail après une longue absence ou après l'âge de 40 ans (LeRAIS, MARIONI, 2004).

\section{Qui sont les femmes au foyer?}

De nombreuses études ont mis en exergue l'augmentation progressive et continue des femmes sur le marché du travail. Les travaux de MARUANI (2006) notamment montrent que ce mouvement est commun aux différents pays européens, même si des différences nationales apparaissent dans son ampleur et sa 
forme. Néanmoins les courbes d'activités des femmes et des hommes ne sont pas identiques et nombreuses sont les femmes qui se retirent de l'emploi pour une période plus ou moins longue. D'après les différentes statistiques consultées, on peut estimer que 15 à $20 \%$ de l'ensemble des femmes se situant dans les tranches d'âge 25-59 ans sont, dans différents pays européens dont la France et la Belgique, des femmes «au foyer», c'est-à-dire des femmes qui ne sont répertoriées comme en emploi ni en recherche d'emploi, même si parfois elles perçoivent des allocations de chômage (GAVRAY, 2008). Les femmes au foyer se retrouvent aussi bien dans la catégorie des femmes non diplômées que diplômées, même si celles-ci sont proportionnellement moins nombreuses (GAVRAY, 2008; Maruani, 2006). Les études sur les trajectoires professionnelles (Remillon, De LARQUIER, 2008) montrent aussi les multiples tentatives de concilier de manière satisfaisante vie familiale et professionnelle (Garner, Meda, Senik, 2005; Tremblay, 2005 ; VENDRAMIN, 2007) et la nécessité d'avoir des revenus suffisants pour subvenir aux besoins familiaux.

\section{Pourquoi se retirer du marché du travail?}

Toutes les enquêtes de budgets-temps mettent en lumière le fait que les femmes restent majoritairement en charge des activités domestiques et éducatives. La spécialisation des rôles au sein des couples est encore largementrépandue, malgré des évolutions ces dernières décennies (GARNER et al., 2005; MEdA, 2001). Les difficultés de conciliation des temps de vie sont mises en avant comme le facteur principal de retrait dans de nombreuses recherches (FAGNANI, 2001 ; HANTRAIS, LeTABliER, 1996; LOLLIVIER, 2005; Meda, Simon, WierinK, 2003). La flexibilité accrue des emplois renforce ces difficultés de conciliation dans une série de secteurs par ailleurs majoritairement féminins (distribution, nettoyage, restauration, travail en usine) loin des grandes entreprises classiques offrant des horaires plus compatibles avec la vie parentale et plus prévisibles (CORNET, 2005). Les femmes ont donc plus souvent que les hommes un calcul d'opportunité à réaliser quant à leur maintien sur le marché du travail. Il s'avère que les femmes les plus nombreuses à se retirer du marché du travail sont les employées peu diplômées et les ouvrières, ce qui est logique dans une optique d'arbitrage des ressources puisqu'elles perçoivent des salaires moins élevés que les femmes diplômées (Algava, BRESSE, 2005). Ceci est d'autant plus vrai quand il existe des revenus de remplacement qui ne sont pas proportionnels au salaire (comme l'allocation parentale d'éducation en France et le congé parental en Belgique (1)) ou des mesures d'interruption temporaire de travail offrant la possibilité de retrouver son emploi par la suite (pause-carrière en Belgique).

(1) Congé d'une durée très différente: trois ans en France, trois mois en Belgique.
On constate toutefois que des femmes diplômées se retirent également du marché du travail, phénomène qui serait en augmentation ces dernières années, de manière plus marquée aux USA qu'en France et en Belgique (GaVray, 2008; MaIson, 2007). Certains hommes se retirent aussi temporairement du travail pour s'occuper des tâches familiales et parentales, mais généralement dans des couples où ils gagnent moins que leurs femmes (MERLA, 2006). Néanmoins, comme le montre Dominique MAISON (2007), le calcul financier ne suffit pas à expliquer le retrait du marché du travail des femmes : ce calcul se combine avec des conditions défavorables sur le plan professionnel et une vision particulière du rôle de la mère au sein du couple, avec une grande importance donnée au bien-être familial et à l'éducation des enfants. D'autres font ce choix parce qu'elles ont un enfant présentant des problèmes de santé majeurs ou des difficultés scolaires importantes, requérant une attention et des soins difficilement externalisables, mais aussi parfois parce qu'elles doivent prodiguer des soins à des parents ou beaux-parents âgés, à des personnes dépendantes comme des adultes handicapés, ou pour prendre en charge leurs petits-enfants (MÉDA et al., 2003).

\section{Que font les femmes au foyer?}

Il existe peu d'études sur l'organisation des journées des femmes au foyer et leurs activités, un peu comme si le fait d'avoir dit qu'elles étaient «au foyer» et qu'elles s'investissaient dans les tâches domestiques et parentales suffisait à décrire la totalité de leur quotidien.

Les enquêtes budgets-temps montrent que le temps dégagé par ces femmes par rapport à celles qui travaillent est investi prioritairement dans des activités parentales (avec un écart de plus de 10 points). Le temps consacré aux tâches domestiques augmente dans une proportion plus ou moins similaire (9 points); par contre, il est effectué en semaine et non le week-end. Le travail domestique et parental est longtemps resté un «travail invisible», peu pris en compte par le monde politique, économique et scientifique. Toutefois, certains auteurs ont essayé d'établir la valeur des activités de production domestique. De manière générale, toutes les tâches «externalisables» et pouvant être prises en charge le cas échéant par un tiers ont été comptabilisées, qu'il s'agisse des tâches domestiques au sens propre (entretien du linge, préparation des repas, entretien de la maison, courses, etc.), ou des tâches parentales (soins aux enfants, jeux, éducation, covoiturage). L'apport de la production domestique est ainsi estimé de 28 à $44 \%$ du PIB (Chadeau, FouQuet, 1981; Dussuet, 1998).

Comme le souligne Dominique Méda (2000): «L'expression "travail domestique" rend mal compte de l'ensemble des activités et responsabilités assumées par les femmes au foyer: elles (les 
femmes au foyer) prennent en charge non seulement les courses, l'entretien de la maison, l'éducation et les soins aux enfants... mais également la sociabilité, la réception d'amis, la conduite des enfants à l'école, les visites chez le médecin, les maladies d'enfants, tâches qui sont souvent invisibles. JeanClaude Kaufmann $(2000,2005)$ a lui aussi contribué à sortir quelque peu le travail domestique de l'ombre en montrant ce qui s'y joue en termes d'identité et de rapports de couple.

Les études budgets-temps montrent que les femmes au foyer disposent en moyenne de $10 \%$ de temps personnel de plus que les femmes qui travaillent (MAISON, 2007). Ce qu'elles font de ce temps personnel et des temps non comptabilisés dans les catégories précédentes a été fort peu investigué. Nous allons nous intéresser à ces autres activités que prennent en charge ces femmes, à leur raison d'être et à leur articulation avec les tâches parentales et domestiques. Notre intérêt pour ce sujet est né d'une analyse inductive d'interviews réalisées auprès d'une quarantaine de femmes au foyer, qui ont fait émerger ces nombreuses activités «externes» par les méthodes d'analyse du discours. Il nous semble intéressant de les rendre visibles et de discuter de l'impact qu'elles sont susceptibles d'avoir sur un retour sur le marché du travail.

\section{Une enquête auprès de femmes au foyer avec un projet de retour à l'emploi}

Nous avons rencontré des femmes qui avaient interrompu totalement leur carrière professionnelle pendant au moins deux ans pour des raisons familiales et qui étaient déjà revenues sur le marché de travail ou avaient un projet de retour sur le marché du travail à court ou moyen terme, en tant que salariées ou indépendantes. Les femmes ayant arrêté leur activité professionnelle pour convenance personnelle ou longue maladie ne rentraient pas dans le périmètre de cette étude, car notre objectif était d'étudier les difficultés spécifiques rencontrées par les femmes dans la conciliation entre vie professionnelle et vie familiale et parentale.

\section{L'enquête: "recruter" des femmes au foyer}

Nous ne disposions pas d'une base de données correspondant à notre groupe-cible en Belgique francophone. Nous avons dès lors décidé de «recruter» nous-mêmes des femmes qui correspondaient à nos critères, en privilégiant plusieurs voies : un article dans un journal à destination des familles, un réseau de femmes et des associations ayant des projets d'insertion socioprofessionnelle. Notons également que le «bouche à oreille» a fonctionné. En un mois, nous avons reçu plus de soixante mails ou appels téléphoniques de femmes nous infor- mant de leur intérêt pour notre étude et acceptant d'être interviewées sur leur trajectoire professionnelle et leur rapport au marché du travail. Nous avons ainsi pu très rapidement constituer un échantillon de quarante femmes choisies sur la base de critères assurant sa diversité: critères d'âge (situé dans la tranche 30-60 ans), de niveau de diplôme ou de qualification, de lieu de vie (rural, urbain ou périurbain), de nombre d'années passées au foyer et de situation familiale (nombre d'enfants, vivant en couple ou non). Notre objectif était en effet d'avoir un échantillon à même de représenter la diversité des réalités des femmes au foyer.

Nous avons choisi de recourir à une méthodologie qualitative de recherche, qui nous semblait la plus pertinente pour pouvoir appréhender de manière approfondie le vécu de ces femmes. Notre objectif était de travailler sur des trajectoires de vie (SAnseau, 2005) et donc de mettre en lumière le parcours professionnel et familial et les différents éléments avancés pour l'expliquer. Cette approche nous semble la plus pertinente pour donner du sens aux comportements, mais aussi pour faire émerger de nouveaux cadres d'analyse et de nouvelles thématiques (Strauss, Corbin, 1998). De fait, les données ainsi récoltées nous ont permis non seulement de confirmer toute une série d'hypothèses formulées a priori, mais également de voir émerger, grâce aux principes de la recherche inductive, les éléments nouveaux qui font l'objet de cet article.

Nous avons réalisé quarante entretiens individuels sous la forme de récit de vie centré sur la vie adulte des femmes rencontrées. Les entretiens ont été menés en face-à-face. Ils étaient structurés autour d'un canevas identique de questions très larges abordant les études suivies, les expériences professionnelles précédant éventuellement la décision de retrait, la décision du retrait, la période passée au foyer, la décision et les stratégies de retour sur le marché du travail.

\section{Un échantillon comportant davantage de femmes diplômées et de plus de $\mathbf{4 0}$ ans}

Le tableau qui suit présente les caractéristiques de l'échantillon:

-14 femmes ont entre 30 et 39 ans, 20 entre 40 et 49 ans, 8 plus de 50 ans ;

-12 ont un diplôme de l'enseignement secondaire, 12 des études supérieures non-universitaires (graduat - baccalauréat), 19 des études universitaires (master);

-3 ont un enfant, 15 en ont deux, 12 ont trois enfants, 11 en ont quatre, 1 a six enfants et 1 autre sept enfants;

- 11 sont divorcées, les autres sont mariées et vivent en couple.

Notre échantillon est diversifié selon ces critères, mais nous devons bien constater que nous avons peu de femmes de moins 40 ans et que l'échantillon 
est composé majoritairement de femmes ayant des diplômes relativement élevés. Nous aurions voulu aussi le diversifier selon l'origine (en visant des cultures ou des parcours migratoires variés), mais nos tentatives n'ont pas été concluantes. Notre population est donc essentiellement composée de femmes belges. À la lumière de ses caractéristiques, il est évident que des études complémentaires portant sur des femmes ayant de plus faible niveau de diplôme ou d'origines ethniques plus diversifiées apporteraient un éclairage utile.

\section{Tableau 1: Caractéristiques de l'échantillon}

\begin{tabular}{|c|c|c|c|c|c|c|c|}
\hline $\begin{array}{c}\mathbf{N}^{\circ} \\
\text { d'identi- } \\
\text { fication }\end{array}$ & Âge & Diplôme initial & $\begin{array}{c}\text { Nombre } \\
\text { enfant }\end{array}$ & État civil & $\begin{array}{l}\text { Âge de } \\
\text { l'arrêt }\end{array}$ & $\begin{array}{l}\text { Âge de la } \\
\text { reprise }\end{array}$ & État actuel \\
\hline 1 & 41 & Secondaire technique & 2 & Mariée & 28 & & En formation \\
\hline 2. & 48 & Graduat/DEUG en ergothérapie & 2 & Séparée & 35 & & Essai obtenir statut artiste \\
\hline 3. & 39 & Secondaire générale & 4 & Divorcée & 18 & 32 & Secrétaire de direction \\
\hline 4. & 57 & Secondaires techniques & 4 & Mariée & 27 & 50 & Formatrice \\
\hline 5. & 48 & Licence/master en romanes & 2 & Mariée & 32 & 47 & Enseignante \\
\hline 6. & 43 & Graduat/DEUG en tourisme & 2 & Mariée & 36 & & \\
\hline 7. & 43 & Secondaires techniques & 4 & Mariée & 24 & & \\
\hline 8. & 41 & Licence/master en informatique & 2 & Mariée & 35 & 41 & Employée ASBL \\
\hline 9. & 43 & Graduat/DEUG en tourisme & 4 & Mariée & 27 & & En formation \\
\hline 10. & 53 & $\begin{array}{l}\text { Graduat/DEUG en éducation } \\
\text { physique }\end{array}$ & 3 & Divorcée & 21 & & En formation \\
\hline 11. & 44 & Infirmière & 2 & Mariée & 24 & & En formation \\
\hline 12. & 42 & $\begin{array}{l}\text { Licence/master en sciences } \\
\text { politiques }\end{array}$ & 6 & Mariée & 25 & & En formation \\
\hline 13. & 42 & Secondaires techniques & 4 & Divorcée & Indéterminé & Indéterminé & $\begin{array}{l}\text { (Vendeuse: devait se } \\
\text { présenter) }\end{array}$ \\
\hline 14. & 38 & Secondaires inférieures & 4 & Div./coh. & Indéterminé & 38 & Employée (aide soignante) \\
\hline 15. & 50 & Licence/master en droit & 3 & Mariée & 48 & & \\
\hline 16. & 38 & Assistante sociale & 3 & Mariée & 34 & & $\begin{array}{l}\text { Pas encore en recherche } \\
\text { active }\end{array}$ \\
\hline 17. & 42 & Licence/master en droit & 4 & Divorcée & 23 & 37 & Employée administrative \\
\hline 18. & 34 & Licence/master en romanes & 2 & Mariée & 31 & & \\
\hline 19. & 45 & Docteur en médecine & 4 & Mariée & 28 & 40 & Indépendante (osthéopathe) \\
\hline 20. & 40 & Secondaires générales & 1 & Célibataire & 35 & & \\
\hline 21. & 35 & Master $-3^{\mathrm{e}}$ cycle en GRH (France) & 4 & Mariée & 26 & & En formation \\
\hline 22. & 36 & Licence/master en communication & 2 & Mariée & 32 & & \\
\hline 23. & 38 & Secondaire & 1 & Divorcée & $24+36$ & $30+?$ & En formation \\
\hline 24. & 36 & $\begin{array}{l}\text { Licence/master en sciences } \\
\text { politique }\end{array}$ & 3 & Mariée & 30 & & \\
\hline 25. & 38 & Licence/master en gestion & 2 & Mariée & 35 & 38 & Formatrice pour adultes \\
\hline 26. & 30 & Ingénieur agronome & 3 & Mariée & 27 & & \\
\hline 27. & 44 & $\begin{array}{l}\text { Licence/master en sciences } \\
\text { politiques }\end{array}$ & 2 & Mariée & 23 & 41 & Bibliothécaire \\
\hline 28. & 42 & Gadué-DEUG bibliothécaire & 2 & Mariée & 30 & & $\begin{array}{l}\text { Fin de formation assistante } \\
\text { de direction }\end{array}$ \\
\hline 29. & 40 & $\begin{array}{l}\text { Secondaire/DEUG logopédie } \\
\text { inachevé }\end{array}$ & 3 & Divorcée & $29+34$ & $31+?$ & \\
\hline 30. & 42 & Licence/master en géologie & 3 & Mariée & 28 & 41 & Indépendante \\
\hline 31. & 38 & Institutrice maternelle & 1 & Mariée & 26 & & \\
\hline 32. & 54 & Secondaire inférieur & 3 & Divorcée & 31 & 45 & Travail administratif \\
\hline 33. & 35 & Licence/master en histoire & 4 & Mariée & 25 & & \\
\hline 34. & 42 & Licence/master en philo. germ. & 3 & Mariée & 40 & & \\
\hline 35. & 44 & Infirmière & 3 & Mariée & 29 & 40 ans & Formatrice en association \\
\hline 36. & 37 & Licence/master sciences politiques & 3 & Mariée & 25 & & \\
\hline 37. & & Graduat/DEUG en secrétariat & 4 & Mariée & 37 & & \\
\hline 38. & 53 & Graduat/DEUG en chimie & 7 & Divorcée & 28 & 47 & Secrétaire \\
\hline 39. & 53 & $1^{\text {re }}$ année d'assistante sociale & 2 & Mariée & 46 & & \\
\hline 40. & 54 & Comptable & 2 & Divorcée & Multi & Multi & Indépendante \\
\hline 41. & 42 & Licence/master en psychologie & 2 & Divorcée & & & \\
\hline 42. & & $\begin{array}{l}\text { Licence/master en germanique/ } \\
\text { gestion }\end{array}$ & 3 & Mariée & 40 & & \\
\hline 43. & 38 & Coiffeuse & 2 & Mariée & $26+30$ & $28+?$ & \\
\hline
\end{tabular}


Les entretiens individuels ont été réalisés en Belgique francophone entre les mois de février et de mai 2008. Nous avons rencontré les femmes dans le lieu de leur choix : à leur domicile, dans un snack-bar, dans les locaux d'une association qu'elles fréquentent. Chaque entretien a duré entre 1 et 3 heures.

\section{Une analyse réalisée en partie avec un logiciel d'analyse lexicale}

Les entretiens ont été enregistrés avec l'accord préalable des femmes rencontrées et ensuite intégralement retranscrits. L'analyse des données a suivi une approche thématique reprenant les thèmes identifiés précédemment dans la revue de littérature, mais aussi inductive, pour faire émerger des sujets et thèmes non prévus initialement. Deux chercheuses ont réalisé l'analyse de manière conjointe en vue de confronter leur point de vue. Outre l'analyse du texte intégral des interviews, nous avons fait appel au logiciel libre d'analyse qualitative Cassandre (2). Conçu pour assister les chercheurs en sciences humaines et sociales, Cassandre est un outil interactif d'analyse lexicale: il permet en effet d'articuler un corpus de textes et des hypothèses de recherche. L'outil se présente comme une plate-forme d'analyse de textes. L'utilisateur identifie, dans ceux-ci, les mots-clés correspondant aux phénomènes qui l'intéressent. Le logiciel identifie tous les passages comprenant ces mots-clés, les passages pouvant ainsi être comparés. Ces comparaisons permettent d'affiner les hypothèses de travail et soulèvent éventuellement de nouvelles questions (LEJEUNE, 2008). Les entretiens ont, à cette fin, été intégralement incorporés dans le logiciel, des mots clé ayant été définis par les chercheuses en fonction des questions initiales de recherche. Ce logiciel a permis de visualiser l'importance de certains thèmes et les liens faits par les femmes de notre échantillon entre plusieurs d'entre eux. In fine, le logiciel a facilité le repérage de citations pertinentes.

\section{Des femmes «inactives" qui se décrivent comme très actives!}

Quand les enfants sont en âge scolaire, et notamment à partir du moment où ils restent à l'école sur le temps de midi, le temps domestique et parental ne «remplit» pas toute la journée des semaines «ordinaires » (c'est-à-dire des semaines sans maladie et congés scolaires). Peu à peu, des plages de temps «libres » se dégagent, plus ou moins importantes selon l'engagement de la femme dans les tâches ménagères (temps variable accordé au nettoyage, à la préparation des repas et au soin du linge selon les goûts, les compétences, les représentations et les attentes fami-

(2) http://cassandre-qda.sourceforge.net/ liales à cet égard). Le temps «libre» dépend aussi des autorisations que les femmes se donnent ou non: certaines jugeront par exemple qu'étant au foyer, elles ont l'obligation d'aller chercher leurs enfants à l'école dès la fin des cours, alors que d'autres estimeront qu' 1 heure ou 2 de garderie ou à l'étude ne sera pas préjudiciable à ceux-ci et leur permettra de développer plus d'activités «externes» au foyer.

Toutes les femmes au foyer que nous avons rencontrées développent différents types d'activité externe. Dans la plupart des cas, elles ne les réalisent pas pour se positionner de manière proactive sur le marché de l'emploi, mais plutôt en raison d'autres logiques d'action que nous analyserons dans la suite de cet article. Néanmoins, ces différentes activités ne sont pas sans lien avec le marché du travail.

Les types d'activités identifiées sont les suivants: - l'investissement dans des activités de bénévolat; - la création artistique;

- la formation;

- le travail non déclaré, occasionnel ou de faible ampleur;

- un processus d'aller-retour sur le marché du travail déclaré.

Nous commencerons par présenter ces différents types d'activité en expliquant à quelles tâches elles correspondent, ainsi que les raisons qui poussent les femmes au foyer à les réaliser. Nous soulignerons également les effets de cet investissement sur elles (notamment, sur leur sentiment de reconnaissance), sur leur famille et sur leur environnement. Ensuite, nous présenterons les logiques d'action qui soustendent ces activités, logiques d'action qui sont en grande partie similaires à celles qui sous-tendent les activités salariées. Enfin, ces logiques d'action sont mises en relation avec différentes variables contextuelles. Cette étape est importante car elle permet de prendre en compte l'hétérogénéité des profils de femmes au foyer et donc des compétences qu'elles ont à offrir sur le marché du travail ainsi que de la diversité des trajectoires: il n'y a pas une seule façon d'être «femme au foyer». Nous terminons en faisant le lien avec la notion d'employabilité et la question de la valorisation des compétences ainsi développées sur le marché du travail.

\section{Les activités bénévoles: un pont entre deux mondes?}

Certaines femmes développent un bénévolat informel et de proximité, vis-à-vis de voisins en difficulté ou de femmes qui travaillent, aidant ces dernières dans leur propre conciliation de leur vie familiale et professionnelle:

«J'aide les mamans qui travaillent et qui ont des problèmes avec leurs enfants. J'ai gardé ici un bébé pendant 1 an. Il y a une autre maman qui a trois garçons: souvent, je les prends, je les amène à l'école. J'ai toujours fait des choses comme ça, pour les aider elles. Parce qu'elles savent que moi je suis là. 
Ou alors, quand il y en a un qui est malade, souvent c'est moi aussi ». (22, 36 ans, master en sciences de la communication, deux enfants, mariée).

Elles s'impliquent aussi dans les structures liées à la vie de leurs enfants: les lieux de rencontre parents-enfants (bébé rencontres), les haltes-garderies, l'école. On retrouve la plupart des femmes interviewées actives au niveau de l'école de leurs enfants. Cet apport est loin d'être négligeable car, outre leur participation aux comités de parents, elles s'investissent dans l'organisation des activités festives (source de revenus pour l'école), elles sont accompagnatrices lors des excursions scolaires, elles tiennent la bibliothèque de l'école, elles proposent des animations sur les temps de midi comme des ateliers de lecture, de dessin, de musique, etc. Les écoles fonctionnent donc en s'appuyant grandement sur cette «main-d'œuvre invisible».

À côté de cet engagement lié aux enfants, plusieurs femmes ont pris des responsabilités ou se sont engagées dans le monde associatif (engagement humanitaire, accompagnement pour les personnes en fin de vie, animation d'une maison de quartier, association de défense de l'environnement, etc. ). Ces activités leur donnent une possibilité de développer de nouvelles compétences (informatique, gestion de réunion, etc. ), leur assurent un réseau social, leur offrent une nouvelle ouverture sur le monde extérieur, leur permettent de développer une vision positive de leur place dans la société.

\begin{abstract}
«Avec cette organisation, je me suis remise, là c'était absolument gratuit, à avoir des réunions plus régulièrement, à devoir faire des comptes rendus de réunions, des trucs comme ça. À ce moment-là, je me suis intéressée à l'ordinateur, j'ai eu une adresse mail. J'ai commencé à me réinvestir dans la vie en société, à m'intéresser à d'autres choses, je veux dire me remettre plus dans le monde, à sortir un peu de ma petite bulle familiale et à me confronter à d'autres ». (9, 43 ans, Graduat/DEUG en tourisme, quatre enfants, mariée).
\end{abstract}

Les femmes qui ont développé des activités bénévoles dans le milieu associatif ont au minimum obtenu leur diplôme du secondaire (elles sont dix-neuf à avoir évoqué un engagement associatif à des degrés divers). Cinq d'entre elles sont actives dans un parti politique et ont été élues locales. Les femmes ne possédant pas de diplôme de secondaire et se trouvant dans des situations financières précaires développent plutôt des activités rémunérées ou du bénévolat de proximité informel. Outre que les activités bénévoles exercées dans un cadre formel sont les plus susceptibles d'être valorisées sur le marché de l'emploi, elles peuvent aussi mener directement à l'emploi: quatre des femmes que nous avons rencontrées ont été engagées par une des associations dans lesquelles elles avaient travaillé bénévolement.

\section{La création artistique: une opportunité de valorisation}

La création artistique peut prendre des formes très différentes selon les cas rencontrés. Cette création peut concerner la fabrication de bijoux, de paysages miniatures, la peinture, la sculpture, le chant, la musique, la danse mais aussi l'aménagement d'intérieur ou de jardin. Ces activités ne sont pas l'apanage exclusif des femmes vivant dans un milieu privilégié, même si les arts les plus reconnus se retrouvent principalement chez les femmes avec les plus hauts niveaux de diplôme (une femme sculptrice, deux femmes peintres, quatre femmes musiciennes, une femme créatrice de bijoux, six d'entre elles ayant un diplôme d'études supérieures). Par cette création, les femmes cherchent à «produire» des œuvres et objets qui soient reconnus, à se créer une identité autre que celle de femme au foyer. Elles sont fortement liées à un moment de plaisir personnel. Il est tout à fait remarquable qu'elles présentent presque toujours ces activités comme accessoires et n'empiétant pas sur la vie familiale. Par ailleurs, même quand l'occupation artistique remplit de 6 à 8 heures de leurs journées ou quand elle débouche sur des expositions, des représentations et des ventes, ces femmes ne se reconnaissent pas le titre d'artiste.

«J'ai suivi des cours de peinture pendant trois ans et puis, j'ai travaillé toute seule pendant les dix années suivantes. 8 heures par jour: à 9 heures du matin je montais dans mon atelier (c'est en fait une petite pièce que l'on a fait faire au-dessus de ma chambre) de 9 heures à 10 heures du matin jusqu'à 15-16 heures, où j'allais rechercher les enfants. Je faisais des expos. J'ai finalement créé un petit bouquin avec mes dix ans de peinture, pour les enfants. En même temps, cela me servait de book. J'ai aussi fait un livre de chroniques que j'ai vendu à cinquante exemplaires, ce n'est pas énorme mais... Donc c'est finalement à partir du moment où j'ai arrêté de courir que j'ai commencé à me connaître...». (5, 48 ans, master en langues romanes, deux enfants, mariée).

Peut-être touche-t-on ici à une définition genrée de ce qu'on est et de ce qu'on fait marquée par le genre: une femme qui se consacre quelques heures tous les jours à la peinture tout en assumant la gestion quotidienne du foyer se décrit avant tout comme une femme au foyer qui a une activité accessoire et de loisir.

Certaines d'entre elles formulent le projet que ces activités occasionnelles puissent être un tremplin vers un statut d'indépendant. Les recherches que nous avons menées par ailleurs sur l'entrepreneuriat féminin (CORNET, CONSTANTINIDIS, 2004) ont montré que de nombreuses femmes créaient leur activité dans les secteurs de l'artisanat et autres activités commerciales créatives qui étaient le prolongement d'une activité développée à domicile pendant une période passée au foyer. Pour certaines d'entre elles, cela constituera une opportunité de retour sur le 
marché du travail mais beaucoup vont être confrontées à la difficulté de transformer cette activité de loisirs en une activité rentable.

\section{Les formations: une anticipation du retour sur le marché du travail}

Une partie du temps libre est utilisée par certaines pour s'engager dans des formations, plus ou moins longues. Elles font ce choix soit par intérêt personnel, soit pour mieux gérer leur situation familiale (comprendre et accompagner la maladie ou le handicap d'un proche, se défendre dans une procédure de divorce, aider les enfants dans leur parcours scolaire), soit dans la perspective d'un retour ultérieur sur le marché du travail. Ces formations peuvent être de quelques jours ou s'étendre sur plusieurs années, être plus ou moins contraignantes en termes d'horaires, de travail à domicile, de stages et d'examens. Plus l'investissement est important, plus le support familial joue un rôle dans la poursuite de la formation. En effet, certaines formations répondent à des exigences similaires à celles d'un emploi. L'engagement dans cette démarche va donc dépendre de l'âge et du nombre d'enfants, du soutien du conjoint, de l'aide accessible dans le réseau proche. Elle permet parfois de mettre en place une nouvelle distribution des rôles au sein du couple.

"Donc j'ai voulu commencer des études, je me suis dit "je vais attendre que la plus jeune ait au moins fait une année de maternelle" parce que la première année, j'aime bien qu'elle aille le matin à l'école et qu'elle puisse rentrer à midi pour faire la sieste. Donc j'ai commencé mes études quand la petite rentrait en deuxième maternelle. Et j'ai fait mes trois années d'étude, je partais à 7 heures du matin et je revenais le soir. J'ai eu de la chance parce qu'à ce momentlà, mon mari travaillait pour une société à domicile. Donc, le matin, moi je partais avant que les enfants se lèvent et il était là pour assurer, le soir c'était moi». (30, 42 ans, master en géologie, trois enfants, mariée).

Néanmoins certaines femmes continuent à tout assumer de front, préparant, par exemple, les repas à l'avance afin que le conjoint et les enfants n'aient qu'à les réchauffer, si les cours ont lieu en soirée ou programmant les transports et la prise en charge des enfants en mobilisant un réseau d'aides externes.

Ces formations sont présentées comme un besoin par les femmes rencontrées car cela leur donne une ouverture vers l'extérieur du ménage et leur apportent, en général, un regain de confiance en elles. Elles ont également un effet positif sur le regard porté sur elles par leurs proches, notamment leurs enfants.

\section{Le travail occasionnel: avoir de l'argent "à soi "}

Chez les ménages modestes, le développement des activités rémunérées occasionnelles va provenir de la volonté de «mettre du beurre dans les épinards» ou de pouvoir «gâter les enfants».
On trouve aussi un manque de reconnaissance du travail de la femme au sein du foyer par le mari et donc une volonté de lui montrer qu'elles peuvent contribuer aussi aux revenus du ménage.

Chez certaines, l'enjeu est de financer certains de leurs besoins personnels. Les femmes que nous avons rencontrées se sont rarement posées la question de leur autonomie financière personnelle au moment du retrait. Cette question est en revanche apparue chez toutes les interviewées dès qu'elles ont voulu développer des activités «pour elles».

«'avais envie de payer moi-même mes cours de
peinture. Tout de suite ça c'est mis, j'ai trouvé une
vieille dame qui cherchait quelqu'un, qui n'était pas
en fin de vie mais qui avait envie un peu de sortir et
donc en fait, je lui faisais ses courses et on allait à
des concerts toutes les deux et au restaurant. Ca lui
a vraiment fait plaisir. Elle était handicapée mais moi
je prenais tout mon temps, j'avais décidé de lui donner
mon temps et elle me payait... J'avais vraiment envie
d'assumer financièrement mes cours de peinture et
donc je me suis dit que j'allais trouver un petit boulot
et ça s'est bien mis ». ( 9,43 ans, bac +2 en tourisme,
quatre enfants, mariée).

Certaines pensent à cette solution pour pouvoir déléguer les tâches qu'elles estiment devoir en principe assumer parce qu'elles sont au foyer. Le fait de trouver l'argent pour payer ces services leur permet de mieux gérer la culpabilité de ne pas tout faire soi-même. L'extrait suivant, tout particulièrement son début, illustre bien ce schéma:

«Je vais dire une bêtise mais... j'aimerais bien avoir quelqu'un qui nettoie chez moi mais je ne peux pas me le permettre parce que j'estime que l'argent doit d'abord servir aux enfants et comme je suis à la maison, voilà. Je me dis que j'aurais ne fut-ce qu'un peu d'argent pour avoir quelqu'un qui m'aide, pour les tâches qui me pompent l'air... ». (12, 42 ans, master de sciences politiques, six enfants, mariée).

Les tensions dans le couple poussent aussi des femmes à se garantir un minimum d'autonomie financière et à anticiper un éventuel retour sur le marché du travail, conséquence d'une séparation.

Les femmes non diplômées sont souvent femmes de ménage chez des connaissances ou des voisines ou effectuent quelques heures de travail dans le secteur de la restauration. La vente à domicile d'objets ménagers, bijoux, vêtements, confitures et tartes maison est une activité qui se retrouve dans les différentes catégories sociales. Il s'agit des activités créatives évoquées ci-dessus, mais elles sont ces fois transformées partiellement en activité commerciale, le plus souvent non déclarées; elles ne débouchent dès lors sur aucun statut. Quand elles sont déclarées, c'est parfois au nom du mari qui prend un statut d'indépendant à titre complémentaire, statut que les femmes ne peuvent prendre du fait qu'elles sont sans emploi et/ou bénéficiaire d'allocations de chômage. C'est, pour certaines d'entre elles, une façon de 
tester l'opportunité d'en faire une activité commerciale avec un statut d'indépendant à titre complémentaire ou principal «un peu plus tard». Toutefois si plusieurs femmes interviewées ont envisagé de se lancer comme indépendantes, très peu a franchi le cap, effrayé par les frais et démarches liés au statut et à l'investissement que cela supposait; le travail au noir est perçu de manière paradoxale comme moins risqué. D'autres donnent quelques heures de cours particuliers ou via une association sans but lucratif, accueillent des enfants ou prennent en charge une personne âgée ou handicapée, etc. Certaines autres organisent des stages payants pour les enfants pendant les vacances scolaires.

Ces activités occasionnelles, outre leur apport financier, sont souvent présentées comme une ouverture sur le monde extérieur. Elles choisissent souvent des activités rémunérées occasionnelles dans lesquelles elles trouvent du plaisir et une reconnaissance leur conférant un sentiment d'utilité sociale et leur permettant de conserver une vie sociale. Ces activités contribuent aussi à leur donner une identité «d'active» auprès de leurs proches et à leurs propres yeux.

Un fait marquant est l'importance donnée au fait que ces activités ne doivent pas gêner l'organisation familiale. Elles s'organisent dans les zones de liberté: pendant les temps scolaires ou en soirée, quand les enfants sont au lit et que les tâches parentales et familiales sont prises en charge. Elles choisissent des activités où elles peuvent avoir une certaine autonomie sur la gestion de leur horaire de travail et, dans une certaine mesure, les conditions d'exercice de celui-ci. Ce résultat rejoint celui des travaux de DéBet et Veretout (2001), qui montrent que le travail au noir, outre l'exploitation et l'insécurité qui lui sont liés, permet (aussi) d'échapper à certains aspects de la condition salariale et qu'il rejoint parfois les vieux rêves de l'autonomie ouvrière et du travail libéré.

Pour un retour dans un emploi salarié, ces compétences sont difficiles à valoriser: en effet, comme il s'agit souvent de travail en noir, les femmes n'osent pas les valoriser dans leur dialogue avec les organismes d'insertion professionnelle quand elles retournent sur le marché de l'emploi et hésitent à le faire figurer dans leur $\mathrm{CV}$.

\section{Les allers-retours sur le marché du travail formel}

Les besoins financiers sont parfois plus aigus ou l'envie de travailler plus impérieuse. Les femmes cherchent alors des solutions pour retourner sur le marché du travail, les moins qualifiées se heurtent cependant à des offres d'emplois précaires ou qui ne sont pas adaptées à leur vie familiale. De petits boulots en petits boulots, elles peuvent finir par retourner au travail occasionnel non déclaré, perçu comme ayant moins de contraintes et laissant plus d'autonomie.

"J'ai recontacté une dame qui organisait des showrooms. Elle avait besoin de deux saisons de mannequins. On était très serrés financièrement et j'avais la taille qu'il fallait. J'ai fait les deux saisons et cela complétait nos revenus. [...] Puis j'ai été enceinte et donc j'ai arrêté le travail de mannequin évidemment. Je suis retournée au chômage mais j'ai trouvé un travail de délégué pour des vêtements pour enfants. Les jours où je faisais des ventes privées, je noircissais la case sur la carte du chômage. Cela a mis un peu d'eau au moulin. Au début cela marchait très bien [...] mais suite à un changement de politique commerciale, cela n'a plus marché, alors j'ai arrêté. Après, j'ai fait des ménages (au noir), je ne vais pas vous le cacher...». (13, 42 ans, secondaire technique, quatre enfants, divorcée).

Beaucoup de ces retours se soldent par un échec: difficultés de concilier travail et obligations familiales, problèmes de transport, accidents familiaux, contrats précaires, conditions de travail défavorables qui les font hésiter à postuler pour un nouvel emploi.

«J'ai fait l'expérience d'assistante du directeur $d u$ personnel pendant quatre ans. J'ai eu ma deuxième fille pendant ce temps-là. C'était une société familiale qui s'essoufflait un peu et la patronne voulait faire rentrer sa fille dans la société. Donc, elle voulait me mettre dehors gentiment. Moi cela m'arrangeait parce que j'étais enceinte de la troisième, mon mari allait partir en mission à l'étranger. Je n'avais toujours pas mon permis de conduire. Donc je me suis dit: cela tombe bien, je vais avoir la troisième et après je recommencerai à travailler. Seulement du jour au lendemain, mon mari m'a quittée... ». (29, bac + DEUG logopédie inachevé, trois enfants, divorcée).

Ces allers-retours permettent aux femmes concernées de rester en prise avec le marché du travail, de maintenir des compétences et de conserver ou récupérer une série de droits (notamment en tant que chômeuses), d'avoir un revenu. Mais on a l'impression que c'est plutôt une contrainte qu'un choix.

Certaines aussi rencontrent de réelles difficultés à se remettre au rythme d'un emploi et à ses contraintes, les allers et retours sont le signe de ces difficultés et de ce malaise.

«Moi c'est un peu particulier puisqu'en dix ans, j'ai eu des longues pauses de chômage qui n'ont pas toujours été intentionnelles mais pendant lesquelles je n'ai pas non plus cherché pour reprendre du travail. Je me suis retrouvée au chômage et je me suis dit que j’allais en profiter. Pendant ces dix années-là, j'ai eu chaque fois des périodes de deux ou trois ans de chômage avant de reprendre une recherche active de travail. La première fois, je croyais vraiment avoir trouvé mon emploi idéal parce que j'adore le commerce et je n'avais plus envie d'être indépendante, je trouvais que c'était trop de risques en étant seule donc j'avais 
trouvé une place de gérante, j'étais donc employée. En fait, je bénéficiais d'un plan d'aide à l'emploi, donc ils m'ont engagée pendant deux périodes de six mois, et quand les employeurs n'ont plus eu droit aux réductions, ils n'ont pas renouvelé mon contrat donc je me suis retrouvée de nouveau au chômage. Quelque temps après, un an je crois, j'ai retrouvé encore un travail d'employé et je n'ai plus supporté la pression. Pendant toute cette période-là, le monde du travail a vraiment changé et je ne supporte pas la pression et la façon de travailler actuelle ». (40, 54 ans, bac +3 , deux enfants, divorcée).

On voit que ces femmes développent difficilement un projet professionnel en partie parce que les réalités familiales continuent de primer, mais aussi parce que le marché du travail est marqué par une précarité dont elles ont été victimes.

\section{L'analyse des rationalités qui sous-tendent ces activités}

Nous avons listé différentes activités externes réalisées par les femmes au foyer. Il s'agit maintenant suivant une approche compréhensive d'apporter un éclairage sur les rationalités qui sont à l'œuvre derrière la mise en place de ces activités (approche compréhensive). Ces logiques d'action ne dépendent pas seulement des acteurs mais aussi du contexte qui structure le comportement, au travers de contraintes objectives et subjectives.

\section{Des objectifs multiples}

Une logique d'action créative. Une partie des femmes interrogées exprime la volonté de «faire des choses» qui ont une valeur ajoutée, qui démontre un talent, des compétences et un travail personnel. Ceci regroupe toutes les activités artisanales et artistiques, mais aussi une partie des activités occasionnelles plus classiques (vendre, mais de façon originale en organisant des défilés chez soi, par exemple, ou en créant un site internet). On sent ici une volonté de reconnaissance au-delà du rôle de mère et d'épouse.

Une logique d'action de plaisir personnel, de réalisation de soi (du «temps à soi et pour soi»). La journée d'une femme au foyer est fortement orientée vers le service aux autres: la famille mais aussi les grands-parents, le voisinage, les parents d'autres enfants qui travaillent. Plusieurs formulent l'envie d'avoir un temps «pour soi et à soi », hors de la logique de service aux autres. Cela se traduit au travers de toutes les activités créatives mais aussi du bénévolat, de l'engagement politique, des formations. On sent aussi dans certaines interviews que si ce temps est souhaité, les femmes ne se l'autorisent que sous certaines conditions : que cela ne perturbe pas la vie familiale et parentale, que cela ne grève pas les revenus du ménage.
Une logique de recherche de liens sociaux, d'ouverture «externe». Bon nombre des femmes rencontrées ne se sentent pas suffisamment insérées socialement au travers de leur vie familiale et amicale. Le fait de suivre des formations, de s'investir dans du bénévolat, de développer une activité semi-professionnelle leur permet de sortir d'un certain isolement et de s'insérer dans des réseaux. Cette recherche de contacts se fait dans une logique affective et sociale et parfois, dans l'idée de maintenir ou créer des contacts qui pourront faciliter le retour sur le marché du travail.

Une logique de recherche d'utilité sociale au-delà $d u$ foyer. Cette logique révèle un souci de se rendre «utile» à la société en réalisant des activités d'aide et de soutien au-delà du foyer. Le simple « dévouement» à la famille n'est pas jugé suffisant aux yeux de plusieurs femmes, qui ne trouvent pas suffisamment de valorisation dans le travail domestique. On retrouvera cette logique d'action à l'œuvre dans bon nombre d'activités bénévoles mais aussi dans le travail occasionnel tel que l'accueil d'enfants, l'accompagnement de personnes âgées ou handicapées, etc. Une logique de formation professionnelle. Cette logique se retrouve principalement dans la poursuite de formations mais aussi par l'exercice d'activités occasionnelles qualifiantes et des allerretours voulus sur le marché de l'emploi, par une remise à jour des connaissances dans le domaine de la formation initiale ou dans le secteur d'une activité professionnelle antérieure, par un investissement créatif ou artisanal à finalité professionnelle. Cette logique d'action est la plus proche d'une stratégie visant au retour sur le marché de l'emploi.

Une logique d'action "financière». Il s'agit, par l'activité développée, d'assurer des revenus complémentaires à la famille ou de rémunérer personnellement ses dépenses personnelles. Dans ce cas, il n'y a pas toujours pression financière «objective» mais subjectivement, la femme ne veut pas «devoir» à son mari le financement de ses loisirs ou ne veut pas prélever cette part sur le revenu du ménage, indiquant en quelque sorte par là qu'elle ne reconnaît pas suffisamment de valeur financière au travail qu'elle accomplit au sein du foyer pour pouvoir en dégager un bénéfice personnel.En nous référant à l'étude de BAUDELot et Gollac sur le bonheur au travail (2003), nous pouvons constater que les sources de satisfaction que recherchent les femmes au foyer dans leurs diverses activités externes sont finalement fort similaires à celles que recherchent les travailleurs: recherche de contacts sociaux, «faire-créer», «s'enrichir personnellement», «servir-aider».

\section{Des différences mais aussi beaucoup de similarités}

Nous avions volontairement cherché à délimiter un échantillon hétérogène de femmes au foyer. 
L'hypothèse était que les logiques d'action seraient différentes selon ces différents critères (âge, niveau de diplôme, nombre d'enfants à charge). Cette hypothèse n'est pas vérifiée. En effet, malgré la grande hétérogénéité des femmes rencontrées, nous avons pu identifier avec constance la présence de ces différentes logiques d'action chez toutes les femmes rencontrées. Par contre, c'est dans la mise en œuvre de ces logiques et dans le degré d'importance et d'investissement accordé à chacune de d'entre elles que les différences apparaissent.

Le niveau de qualification est incontestablement un élément majeur de différenciation de notre échantillon. Il guide le type d'activités que les femmes au foyer entreprennent et notamment l'investissement important dans le bénévolat formel ou les activités artistiques. Ainsi, la dimension «plaisir et épanouissement personnel» ne se retrouvera pas dans la même mesure chez les femmes moins diplômées. Pour ces femmes, la logique de plaisir et d'épanouissement personnel sera moins liée à une activité extérieure. Il s'agit plutôt d'avoir du plaisir et une reconnaissance autour d'une activité domestique (plaisir de faire un bon repas, plaisir de jardiner ou d'entretenir un potager).

Les conditions de vie jouent également un rôle. Les femmes les moins diplômées sont celles qui vont le plus souvent faire des allers et retours vers le marché de l'emploi et tenter de trouver des activités rémunérées pour augmenter les revenus globaux du ménage (moins pour leur besoin personnel). Mais les femmes diplômées peuvent aussi mettre en avant ces logiques de survie et de niveau de revenus quand le couple est en crise et qu'elles vivent ou ont vécu une séparation.

La séparation du couple met souvent les femmes en action et les poussent à investir dans de nouvelles activités: formation professionnelle, recherche d'activités rémunérées. Elles mettent toutes aussi en avant l'importance «maintenant» de penser à elle et plus seulement aux autres. Certaines profitent des crises dans le couple pour obtenir un rééquilibrage des différentes activités et un nouveau partage des tâches domestiques et parentales avec presque toujours l'obtention d'un «temps pour soi», non négocié quelques années auparavant.

Le nombre d'enfants, les âges des enfants, l'écart d'âge entre les enfants sont autant d'éléments qui vont jouer un rôle important dans l'investissement des femmes au foyer car ils vont déterminer le temps que les femmes peuvent consacrer à des activités externes au foyer. La charge d'enfants en bas âge (avant l'entrée en primaire) freine les femmes par rapport aux différentes activités. Toutefois, nous avons pu constater que les mères de familles nombreuses «lâchent du lest» par rapport à leur cadet: là où elles s'interdisaient des activités externes avec les aînés, elles vont se les permettre avec l'enfant suivant dès que celui-ci entre en maternelle (voire avant), ayant acquis avec l'expérience une certaine sérénité par rapport à la gestion de leurs temps d'absence auprès de leurs enfants.

\section{L'employabilité des femmes au foyer: bien réelle et pourtant peu visible}

Notre recherche s'intéressait aux femmes qui veulent retourner sur le marché du travail ou qui viennent de retrouver un emploi. Ce qui nous a frappées au terme des interviews est le peu de mobilisation dans la recherche d'emploi, par les femmes et leurs accompagnateurs, des compétences acquises pendant leur période de retrait du marché du travail. Pourtant, il nous semblait que ces activités étaient susceptibles d'être valorisées sous la forme de compétences et de capacités d'adaptation personnelles mobilisables pour la recherche d'emploi. C'est un peu comme si les seules compétences valorisées étaient les tâches parentales et familiales avec, pour les moins diplômées d'entre elles, un retour presque prédéterminé dans des métiers de services de proximité (garde d'enfants, nettoyage et soins aux personnes).

Les entretiens ont mis en évidence la difficulté de mettre en avant ces compétences acquises. Cette difficulté est en réalité double: d'une part, les femmes elles-mêmes ne sont pas conscientes que leurs compétences peuvent être valorisées sur le marché de l'emploi, parce qu'officiellement les activités qui leur ont permis d'acquérir ces compétences ne sont pas reconnues ni comptabilisées (par exemple, en matière d'organisation d'événements comme les fêtes à l'école de leurs enfants). D'autre part, l'entourage, les conseillers en orientation professionnelle ainsi que les employeurs ont tendance à faire peu de cas d'un CV construit au départ sur des compétences acquises en dehors du marché du travail formel. Une telle attitude les renvoie dès lors au diplôme qu'elles ont acquis, il y a parfois plus de vingt ans, à un métier qu'elles n'ont plus exercé depuis longtemps, voire jamais. Pourtant, les femmes au foyer qui ont retrouvé du travail soulignent que ce n'est pas sur la base de leur diplôme initial qu'elles peuvent le mieux «se vendre» sur le marché du travail, même si celui-ci assure un certain «niveau » et peut être un prérequis. En effet, elles sont alors en concurrence avec des jeunes ayant le même diplôme mais qu'elles ont obtenu beaucoup plus récemment, voire un diplôme plus élevé, ainsi qu'éventuellement une expérience professionnelle récente. Par contre, celles qui ont eu le réflexe de mettre en évidence les compétences acquises pendant leur période au foyer ont pu, lorsqu'une rencontre en face à face était possible avec l'employeur, faire la différence.

Ceci nous amène à plaider pour un réel dispositif de validation des compétences auprès des femmes au foyer qui veulent réintégrer le marché du travail. Il pourrait s'articuler autour de différents axes : 
- Les compétences mobilisées dans la sphère familiale: soins de puériculture aux petits enfants, compétences pédagogiques liées aux activités d'éveil et au suivi des devoirs, compétences liées à la confection des repas, dans certains cas aux activités de jardinage et d'entretien d'un potager, compétences liées à la gestion du budget familial.

- Les compétences mobilisées dans la sphère du bénévolat informel: accompagnement de personnes malades ou handicapées, accompagnement des personnes en fin de vie, accueil de l'enfant, etc.

- Les compétences mobilisées dans la sphère du bénévolat formel: tenue de livres de compte, comptabilité, vente, promotion de produits ou de services, bureautique, accueil de personnes en difficulté, accueil de «clients » d'une association, organisation d'événements, organisation ou dispense de formations, etc.

-Les compétences mobilisées dans la sphère des activités occasionnelles: souvent techniques de vente, restauration, aide aux personnes, activités ménagères mais aussi traductions, vente par Internet, création de sites.

- Les compétences mobilisées dans la sphère des activités créatives : activités artistiques, massages et soins du corps, bien-être, informatique (création de sites, utilisation d'outils comme Photoshop, etc.).

- Les compétences mobilisées en tant que conjointe aidante: accueil des clients, comptabilité, bureautique.

Faire le bilan de ces compétences et proposer, du moins pour une partie de celles-ci, des mécanismes de validation qui leur permettraient d'entreprendre une formation complémentaire de manière «allégée» (dans les domaines de l'accueil de l'enfant, de l'enseignement, de la gestion, du secrétariat, des métiers de la restauration, des métiers d'aide aux personnes, notamment) constituerait un tremplin non négligeable vers l'emploi.
$* \quad *$

Les «femmes au foyer» que nous avons rencontrées n'avaient pas ce statut comme projet de vie et ne désiraient pas le conserver. Au-delà des nombreuses activités qu'elles déploient «au foyer», ces femmes répertoriées comme «inactives» au regard du marché de l'emploi s'investissent pendant leur période de retrait du marché du travail dans les écoles, dans les comités de quartier, dans les associations bénévoles. Elles développent également des activités artistiques, de service à autrui ou même de production de biens et services. Autrement dit, les femmes dites «au foyer» sont loin d'être des «inactives », mais sont au contraire extrêmement actives dans la sphère privée bien entendu, mais aussi dans la sphère sociale et dans une sphère que nous pourrions qualifier de «paraprofessionnelle», que notre recherche a contribué à mettre au jour.

Nous pouvons conclure qu'être femme au foyer n'est pas automatiquement être une femme coupée du monde extérieur et du monde du travail en particulier. Les contacts et passerelles existent et les frontières sont poreuses: des femmes au foyer exercent des activités occasionnelles, des activités quasi indépendantes, font des allers-retours sur le marché de l'emploi. En outre, les activités qu'elles développent sont traversées par des logiques d'action qui sont également celles que l'on retrouve dans le monde du travail. Il est donc important de rendre visible ces différentes activités méconnues et de permettre notamment ainsi à celles qui les ont développées de les valoriser sur le marché de l'emploi. Cette démarche de valorisation sera bénéfique tant les femmes concernées et leur famille que pour les entreprises à la recherche de personnes dotées de compétences utiles. 


\section{Bibliographie}

Algava E., Bresse S. (2005), «Les bénéficiaires de l'allocation parentale d'éducation: trajectoires d'activité et retour à l'emploi», Études et Résultats, 399.

Baudelot C., Gollac M. (2003), Travailler pour être heureux? Le bonheur et le travail en France, Paris, Fayard.

Chadeau A., Fouquet A. (1981), Peut-on mesurer le travail domestique? Économie et Statistiques, 135, pp. 29-42.

Connet A. (2005), «Flexibilité du temps de travail: des stratégies différenciées pour les hommes et les femmes?», in De Nanteuil M. \& A. s. 1. d. d. El Akremi (Eds.), collection «Sociétés en changement», La société flexible, Paris, Éditions ERES, pp. 250-266.

Dubet F., Veretout A. (2001), Une «réduction de la rationalité de l'acteur: pourquoi sortir du RMI», Revue française de sociologie, 42(3), pp.407-436.

Dussuet A. (1998), Logiques domestiques. Essai sur les représentations du travail domestique chez les femmes actives de milieu populaire, Paris, L'harmattan.

FAGNANI J. (2001), «L'intégration progressive de la mère qui travaille : trente ans de politique familiale en France», Spirale (18), pp.139-155.

Garner H., Méda D., Senik C. (2005), «Conciliation entre vie professionnelle et vie familiale, les leçons des enquêtes menées auprès des ménage », Travail et Emploi, $\mathrm{n}^{\circ} 102$.

Gavray C. (2008), «Genre, emploi et marché du travail : un tableau contrasté». In Cornet A., Laufer J., Belghiti S. (Eds.), Le genre et la GRH: les défis de l'égalité hommes-femmes, collection «AGRH», Paris, Vuibert.

Hantrais L., Letablier M. T. (1996), Familles, travail et politiques familiales en Europe, Paris, PUF.

KaufMann J.-C. (2000), Le cour à l'ouvrage. Théorie de l'action ménagère, Paris, Pocket Agora.

Kaufmann J.-C. (2005), Casseroles, amour et crises: ce que cuisiner veut dire, Armand Colin

LeJeune C. (2008), «Au fil de l'interprétation. L'apport des registres aux logiciels d'analyse qualitative», Revue Suisse de Sociologie, 34 (3), pp. 593-603.

Lerais F., Marioni P. (2004), «Accroître l'emploi des seniors: entre volontés et difficultés», in Lerais F., Marioni P. (Eds.), Dossier âge et emploi: synthèse des principales données sur l'emploi des seniors, vol. document d'études $n^{\circ} 82$, Paris, DARES.

LOLLIVIER S. (2005), «Les choix d'activité des femmes en couple: une approche longitudinale», Économie et Statistiques 349-350, INSEE.

MAison D. (2007), «Femmes au foyer. Expériences sociales», dossier études CNAF-université de Bordeaux 2 (92).

Maruani M. (2006), Travail et emploi des femmes, Paris, La Découverte.

MéDA D. (2000), «Les femmes peuvent-elles changer la place du travail dans la vie?», Droit Social (5), pp. 463-470.

MÉDA D. (2001), Le temps des femmes. Pour un nouveau partage des rôles collection «Champs», Paris, Flammarion.

Méda D., Simon M.-O., Wierink M. (2003), «Pourquoi certaines femmes s'arrêtent-elles de travailler à la naissance d'un enfant?», Premières Synthèses (29.2).

MerLa L. (2006), Appréhension et présentation de soi et transgression des normes de la division sexuelle $d u$ travail: le cas des pères «au foyer» UCL, Louvain-LaNeuve (B).

Remillon D., De Larquier G. (2008), «Assiste-t-on à une transformation uniforme des carrières vers plus de mobilité? Une exploitation de l'enquête "Histoire de vie"», Travail et Emploi, ${ }^{\circ} 113$ (janvier-mars).

Sanseau P.-Y. (2005), «Les récits de vie comme stratégie d'accès au réel en sciences de gestion: pertinence, positionnement et perspectives d'analyse», Recherches qualitatives 25 (2), pp. 33-57.

Strauss A., Corbin J. (1998), Basics of qualitative research: Techniques and procedures for developing grounded theory (2nd ed.), CA, Sage, Thousand Oaks.

Tremblay D. G. (2005), De la conciliation emploi-famille à une politique des temps sociaux, Montréal, Presses de l'université du Québec.

VAn Regenmortel T., De Cock C., Vandeloo R. (1990), Femmes rentrantes: un groupe à risque pas comme les autres. Synthèse, Leuven, Hiva.

VendRAmin P. (2007), Temps, rythmes de travail et conciliation des temps sociaux, Namur, FTU Namur, Belgique. 